\section{RFICs on polydimethylsiloxane for flexible electronics applications}

T. Wang, H.-C. Chen, P.-L. Huang, Y.-Y. Chiang and S.-S. Lu

RFICs were thinned down and transferred to the flexible substrate by the proposed polydimethylsiloxane-substrate transfer technology (PSTT). Measurement results show the feasibility of the proposed technique, and furthermore prove its ability to reduce the substrate loss in the high frequency range. With PSTT, the quality factor of a 3.5 turns inductor is improved from 9.3 to 13.4 and the noise figure of a $5-6 \mathrm{GHz}$ direct-conversion receiver is reduced by $0.5 \mathrm{~dB}$. The demonstration reveals the potential of PSTT for the flexible electronics in RFICs applications.

Introduction: Recently, flexible electronics has drawn significant attention owing to its variety of applications such as flexible displays, smart tags, and wearable products. To achieve this goal, there have been plenty of approaches discussed and developed [1-4]. Among these approaches, the organic thin film transistor (OTFT) is acknowledged for its superiority in mechanical flexibility, but it suffers from low operating frequency, short lifetime and limitation in circuit integration. Sparing the issues of OTFT manufacturing, a novel SOI substrate transfer technology (SOI-STT) [4] was proposed and was more likely to have transistors with better performances; however, the inevitable buried oxide during the SOI-STT process is unavailable in the standard CMOS technology and therefore limits the prospect of system-on-a-chip in the future. To resolve these difficulties, polydimethylsiloxane (PDMS)-substrate transfer technology (PSTT) is proposed in this Letter. With the similar goal, PSTT is, however, implemented in a very different way, which can be fully compatible with standard CMOS ICs and is easy to use for a variety of applications. The proposed technique is demonstrated with an inductor and an RF receiver. To our knowledge, it is the first CMOS RF receiver on flexible substrate that has been characterised and compared.

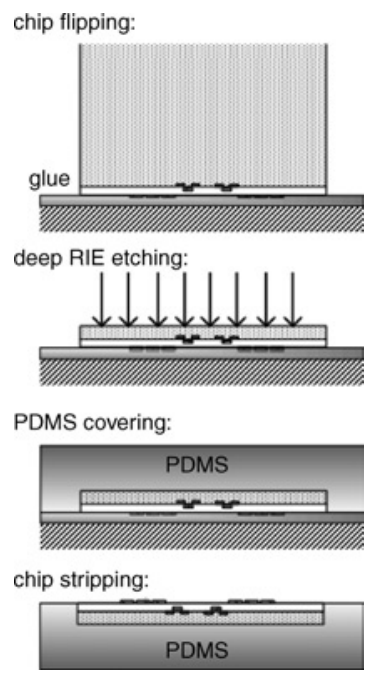

Fig. 1 PSTT processing flow

PSTT and demonstration on RF receiver: The proposed PDMSsubstrate transfer technology (PSTT) is based on jellification of a viscid PDMS layer underneath an ultra-thin substrate. There are four major steps involved in PSTT (see Fig. 1). First, flip the silicon chip and post it on a dummy wafer with thermal dissipative adhesive glued on the chip front-side. The thermal dissipative adhesive was to prevent heat accumulation and possible damage during the following etching step. Secondly, etch the silicon substrate using deep RIE. No wet etching or grinding was involved, making the thinning process stable and safe. The etching rate of deep RIE was approximately $2 \mu \mathrm{m} / \mathrm{min}$, which can be used to estimate the etching time. Thirdly, spread the viscid PDMS on the backside after substrate is thinned down to $20 \mu \mathrm{m}$. The viscid PDMS is able to flow uniformly on the backside surface without any void or seam left, which is of great importance because any unfilled defect will induce the stress accumulation in probing/packaging and may cause the fragile substrate to crack. After spreading the viscid PDMS, a $20 \mathrm{~min} 90^{\circ} \mathrm{C}$ hard bake was continued to jellify the PDMS, forming a flexible cushion underneath the ultrathin substrate. The elasticity of PDMS not only enables the substrate to distribute the contact stress from the chip front-side but also maintains the flexibility of the ultra-thin silicon. The final step is to dip the chip into the acetone to dissolve the adhesive and strip it off from the dummy wafer. Once the chip is stripped, the RF circuits will be clean and ready for test. PSTT is simple to apply in CMOS technology without any process change or additional mask alignements.

To evaluate its feasibility, the proposed PSTT was implemented on an inductor as well as an RF receiver. Fig. 2 shows the experimental results of these RF components/circuits after PSTT. The demonstrated circuits were included in a $5 \times 5 \mathrm{~mm}, 0.18 \mu \mathrm{m}$ CMOS chip, which was thinned and transferred onto the centre of the PDMS substrate bent to show its flexibility.

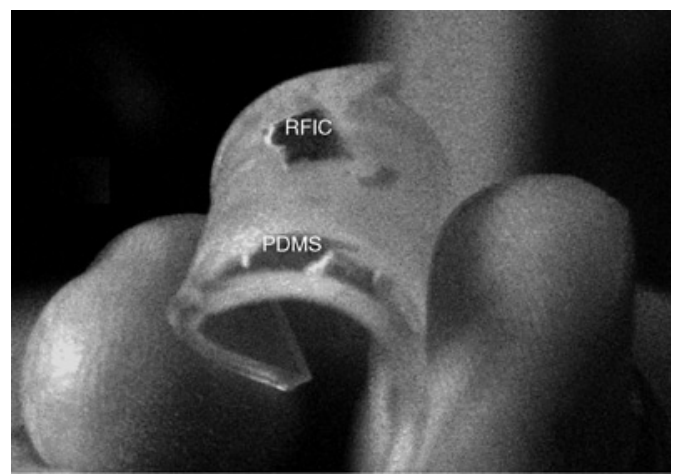

Fig. 2 Flexibility of PSTT chip

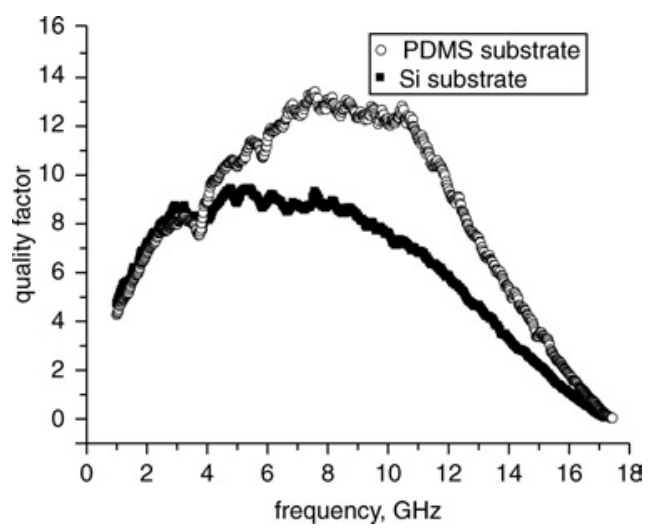

Fig. 3 Quality factor of inductor on silicon and on PDMS

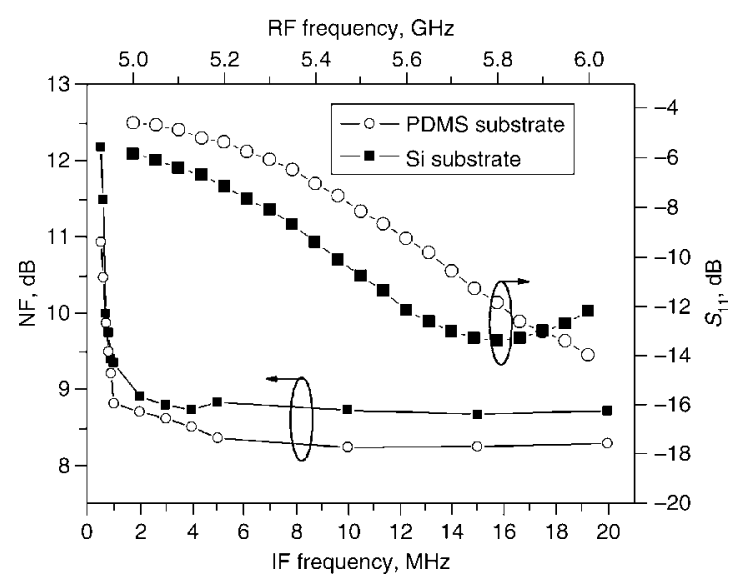

Fig. 4 Measured $S_{11}$ and noise figure of $R F$ receiver

Measurement results and discussion: The quality factor of the 3.5 turns PSTT inductor was extracted from the on-wafer $S$-parameters measurement. It can be seen in Fig. 3 that the peak quality factor is 
improved from 9.3 to 13.4 by PSTT. Such improvement represents a reduction of substrate loss contributed by PDMS substrate. Besides the inductor, a PSTT RF receiver front-end was demonstrated for further examination. The detailed schematic is the same as our previous work [5]. Measurement results of $S_{11}$ and noise figure are shown in Fig. 4. The $S_{11}$ curve is shifted to a higher frequency for the less parasitic capacitance of the thinned substrate and noise figure (NF) is reduced by $0.5 \mathrm{~dB}$ (from 8.7 to $8.2 \mathrm{~dB}$ at $10 \mathrm{MHz}$ offset frequency) for less substrate loss. A summary is given in Table 1. These results have verified the adequacy of PSTT to be implemented on CMOS chips and the low-loss PDMS substrate can even improve the RF performance.

Table 1: Summary of comparison

\begin{tabular}{|l|c|c|c|}
\hline & Parameters & Standard & PSTT \\
\hline \multirow{3}{*}{ Inductor } & Inductance & $2 \mathrm{nH}$ & $2 \mathrm{nH}$ \\
\cline { 2 - 4 } & Quality factor & 9.3 & 13.4 \\
\hline \multirow{4}{*}{ Receiver } & $S_{11}<-10 \mathrm{~dB}$ & $5.45 \mathrm{GHz}$ & $5.65 \mathrm{GHz}$ \\
\cline { 2 - 4 } & Conversion gain & $17.1 \mathrm{~dB}$ & $17.3 \mathrm{~dB}$ \\
\cline { 2 - 4 } & $\mathrm{NF}(10 \mathrm{MHz} \mathrm{IF})$ & $8.7 \mathrm{~dB}$ & $8.2 \mathrm{~dB}$ \\
\hline
\end{tabular}

Conclusion: A CMOS compatible PDMS-substrate transfer technology is proposed which has been proven by demonstration on $0.18 \mu \mathrm{m}$ CMOS RF circuits. This approach has paved the way for CMOS technology towards flexible electronics applications.

(C) The Institution of Engineering and Technology 2007

20 September 2007

Electronics Letters online no: 20072708

doi: 10.1049/el:20072708
T. Wang, H.-C. Chen, P.-L. Huang and S.-S. Lu (Graduate Institute of Electronics Engineering, National Taiwan University, Taipei, Taiwan, Republic of China)

E-mail: sslu@ntu.edu.tw

Y.-Y. Chiang (Institute of Applied Mechanics, National Taiwan University, Taipei, Taiwan, Republic of China)

\section{References}

1 Dimitrakopoulos, C.D., and Mascaro, D.J.: 'Organic thin-film transistors: a review of recent advances', IBM J. Res. Develop., 2001, 45, (1), pp. 11-27

2 Sheraw, C.D., Zhou, L., Huang, J.R., Gundlach, D.J., Jackson, T.N., Kane, M.G., Hill, U.G., Hammond, M.S., Campi, J., Greening, B.K., Francl, J., and West, J.: 'Organic thin-film transistor-driven plolymerdispersed liquid crystal displays on flexible polymeric substrates', Appl. Phys. Lett., 2002, 80, p. 1088

3 Klauk, H., Halik, M., Zschiescahng, U., Eder, F., Schmid, G., and Dehm, C.: 'Pentacene organic transistors and ring oscillators on glass and on flexible polymeric susbtrates', Appl. Phys. Lett., 2003, 82, p. 4175

4 Dekker, R., Dumling, M., Fock, J.-H., Gourhant, O., Jonville, C., Michielsen, T.M., Pohlmann, H., Schnitt, W., and Tombeur, A.M.H.: 'A $10 \mu \mathrm{m}$ thick RF-ID tag for chip-in-paper applications'. Proc. IEEE Bipolar/BiCMOS Circuits and Technology Meet. (BCTM) Tech. Dig., 2005, pp. 18-21

5 Chen, H.-C., Wang, T., and Lu, S.-S.: 'A 5-6 GHz 1-V CMOS directconverstion receiver with an integrated quadrature coupler', IEEE $J$. Solid-State Circuits, 2007, 42, (9), pp. 1963-1975 